\title{
Constraining the level density using fission of lead projectiles
}

J. L. Rodríguez-Sánchez, ${ }^{1}$ J. Benlliure, ${ }^{1}$ H. Álvarez-Pol, ${ }^{1}$ L. Audouin,${ }^{2}$ Y. Ayyad, ${ }^{1, *}$ G. Bélier, ${ }^{3}$ G. Boutoux, ${ }^{3, \dagger}$ E. Casarejos, ${ }^{4}$ A. Chatillon, ${ }^{3}$ D. Cortina-Gil, ${ }^{1}$ T. Gorbinet, ${ }^{3}$ A. Heinz,${ }^{5}$ A. Kelić-Heil, ${ }^{6}$ B. Laurent, ${ }^{3}$ J.-F. Martin, ${ }^{3}$ C. Paradela, ${ }^{1, \ddagger}$ E. Pellereau, ${ }^{3}$ B. Pietras, ${ }^{1}$ D. Ramos, ${ }^{1}$ C. Rodríguez-Tajes,${ }^{1,7}$ D. M. Rossi, ${ }^{6,}{ }^{*}$ H. Simon, ${ }^{6}$ J. Taïeb, ${ }^{3}$ J. Vargas, ${ }^{1}$ and B. Voss ${ }^{6}$

${ }^{1}$ Universidad de Santiago de Compostela, E-15782 Santiago de Compostela, Spain

${ }^{2}$ Institut de Physique Nucléaire d'Orsay, F-91406 Orsay, France

${ }^{3}$ CEA, DAM, DIF, F-91297 Arpajon, France

${ }^{4}$ Universidad de Vigo, E-36200 Vigo, Spain

${ }^{5}$ Chalmers University of Technology, SE-412 96 Gothenburg, Sweden

${ }^{6}$ GSI-Helmholtzzentrum für Schwerionenforschung GmbH, D-64291 Darmstadt, Germany

${ }^{7}$ Grand Accélérateur National d'Ions Lourds, F-14076 Caen Cedex 05, France

(Received 24 August 2015; published 19 October 2015)

\begin{abstract}
The nuclear level density is one of the main ingredients for the statistical description of the fission process. In this work, we propose to constrain the description of this parameter by using fission reactions induced by protons and light ions on ${ }^{208} \mathrm{~Pb}$ at high kinetic energies. The experiment was performed at GSI (Darmstadt), where the combined use of the inverse kinematics technique with an efficient detection setup allowed us to measure the atomic number of the two fission fragments in coincidence. This measurement permitted us to obtain with high precision the partial fission cross sections and the width of the charge distribution as a function of the atomic number of the fissioning system. These data and others previously measured, covering a large range in fissility, are compared to state-of-the-art calculations. The results reveal that total and partial fission cross sections cannot unambiguously constrain the level density at ground-state and saddle-point deformations and additional observables, such as the width of the charge distribution of the final fission fragments, are required.
\end{abstract}

DOI: 10.1103/PhysRevC.92.044612

PACS number(s): 21.10.Ma, 24.10.-i, 25.85.Ge

\section{INTRODUCTION}

A complete description of the fission process still represents a challenge, despite the recent progress based on microscopic quantum dynamical [1-3] and time-dependent Hartree-Fock models [4,5]. Statistical models, proposed soon after the discovery of fission [6], provide a tool to describe fission probabilities at excitation energies around the fission barrier. This approach is justified because, under such conditions, statistical times dominate over the typical timescales for the coupling between intrinsic and collective degrees of freedom $\left(\sim 10^{-21} \mathrm{~s}^{-1}\right)$. At high excitation energies, pre- and postscission particle emission [7-9] and fission probabilities [10-13] indicate that simple statistical approaches are not valid anymore and models describing the dynamics of the process are required [14]. Even in this case, statistical approaches including a time-dependent fission decay width that mimics the evolution of the fission flux across the barrier [15,16], as described by transport equations (e.g., Fokker-Planck or Langevin) including dissipative and stochastic terms [17-20], provide a good description of the fission rate [16]. Statistical approaches are also used to describe the mass and charge distributions of the fission fragments, using a phenomenological description of the mass-asymmetry potential energy [21],

\footnotetext{
*Present address: National Superconducting Cyclotron Laboratory, Michigan State University, East Lansing, Michigan 48824-1321, USA.

†Present address: Université Bordeaux, F-33405 Talence, France.

${ }^{\ddagger}$ Present address: EC-JRC, Institute for Reference Materials and Measurements, Retieseweg 111, B-2440 Geel, Belgium.
}

and the observed broadening of these distributions with the excitation energy of the fissioning system [22-24].

Reliable statistical calculations require an accurate description of the potential-energy landscape and level densities; in particular at ground-state and saddle-point deformations. Fission barriers can be calculated reasonably well by using macroscopic [25] or microscopic [26] approaches. Level densities can be constrained at ground-state deformation by using neutron-induced resonances [27]; however, no direct experimental validation exists for the deformed nucleus at the saddle point. A description of the level density based on the Fermi gas model [28] can yield reasonable results provided that a realistic description of the level-density parameter is used $[23,29,30]$. In the present context, volume and shape dependencies of this parameter are particularly important to correctly describe the level density at saddle-point deformation [31-34].

Despite this rather global agreement on the description of the level-density parameter, several recent works propose empirical modifications of this parameter in order to describe fission rates $[35,36]$. These authors claim that fission rates can be described, even at high excitation energies, without considering any transient time for fission. This conclusion contradicts the well-established role of dissipative effects in the ground-to-saddle fission dynamics to explain pre- and postscission neutron multiplicities [7], $\gamma$-ray emission [8], multiplicities of charged particles [9], as well as fission and evaporation residue cross sections [10-13].

The origin for such contradictory conclusions could be that an effective reduction of the level-density parameter at the saddle point with respect to the ground-state value could mimic 
the fission hindrance induced by dissipative effects. Therefore, many authors claim that conclusions based only on the use of fission rates to constrain the different parameters contributing to the description of this process may lead to ambiguous results [10,23,29,30,37,38].

In order to contribute to this discussion in this work, we take advantage of the complete characterization of both fragments produced in proton-induced fission reactions on ${ }^{208} \mathrm{~Pb}$ recently investigated with the SOFIA setup at GSI $[39,40]$. The inverse kinematics used in this experiment gave us access for the first time to the determination of the velocities, atomic numbers, and mass numbers of both fission fragments. With these measurements we can define observables constraining the role of the level-density parameter in these reactions. Moreover, the relatively high fission barriers of the fissioning nuclei produced in these reactions also contribute, minimizing the impact of collective excitations in the level densities and enhancing dissipative effects.

\section{EXPERIMENT}

Spallation and fragmentation reactions of ${ }^{208} \mathrm{~Pb}$ provide us an excellent tool for investigating level densities together with dissipative effects. As discussed in the pioneering work of Grangé and collaborators [17], the optimal conditions for investigating low-deformation dissipative and transient effects in fission are the use of spherical fissioning systems with low angular momentum and high excitation energies $\left(E^{*}>100 \mathrm{MeV}\right)$. Fortunately, these conditions also represent a good approach to study the effect of the level densities on the experimental observables, such as the fission cross sections, partial fission cross sections, and width of the atomic-number distribution of the fission fragments, because the effect of the rotational and vibrational excitations on the level densities washes out at excitation energies $\sim 40 \mathrm{MeV}[46,47]$. For this purpose, the reactions ${ }^{208} \mathrm{~Pb}(370 A, 500 A$, and $650 A \mathrm{MeV})+p$ and ${ }^{208} \mathrm{~Pb}(500 A \mathrm{MeV})+{ }^{27} \mathrm{Al}$ were selected to investigate the evolution of the previous observables with each energy and entrance channel.

The experiment was performed by the SOFIA collaboration at the GSI facilities in Darmstadt (Germany), where the SIS18 synchrotron was used to accelerate ions of ${ }^{208} \mathrm{~Pb}$ up to $650 \mathrm{~A} \mathrm{MeV}$ with an intensity around $10^{5}$ ions/s. The primary beam was then guided to the experimental setup where fission reactions were induced in different targets. A cylindrical target filled with liquid hydrogen $\left(\sim 85 \mathrm{mg} / \mathrm{cm}^{2}\right)$ was used to investigate spallation-induced fission, while fragmentationinduced fission was investigated by using an aluminium target $\left(\sim 1080 \mathrm{mg} / \mathrm{cm}^{2}\right)$.

The use of the inverse kinematics technique allowed us to separate fission from other reaction channels and also facilitates the identification of both fission fragments $[13,41,42]$. Fission events were identified by using energyloss measurements performed with a double multisampling ionization chamber (twin MUSIC) [43-45], providing the atomic number of the two fission fragments with a resolution better than 0.43 charge units full width at half maximum (FWHM) [42]. In addition, the tracking capabilities of this
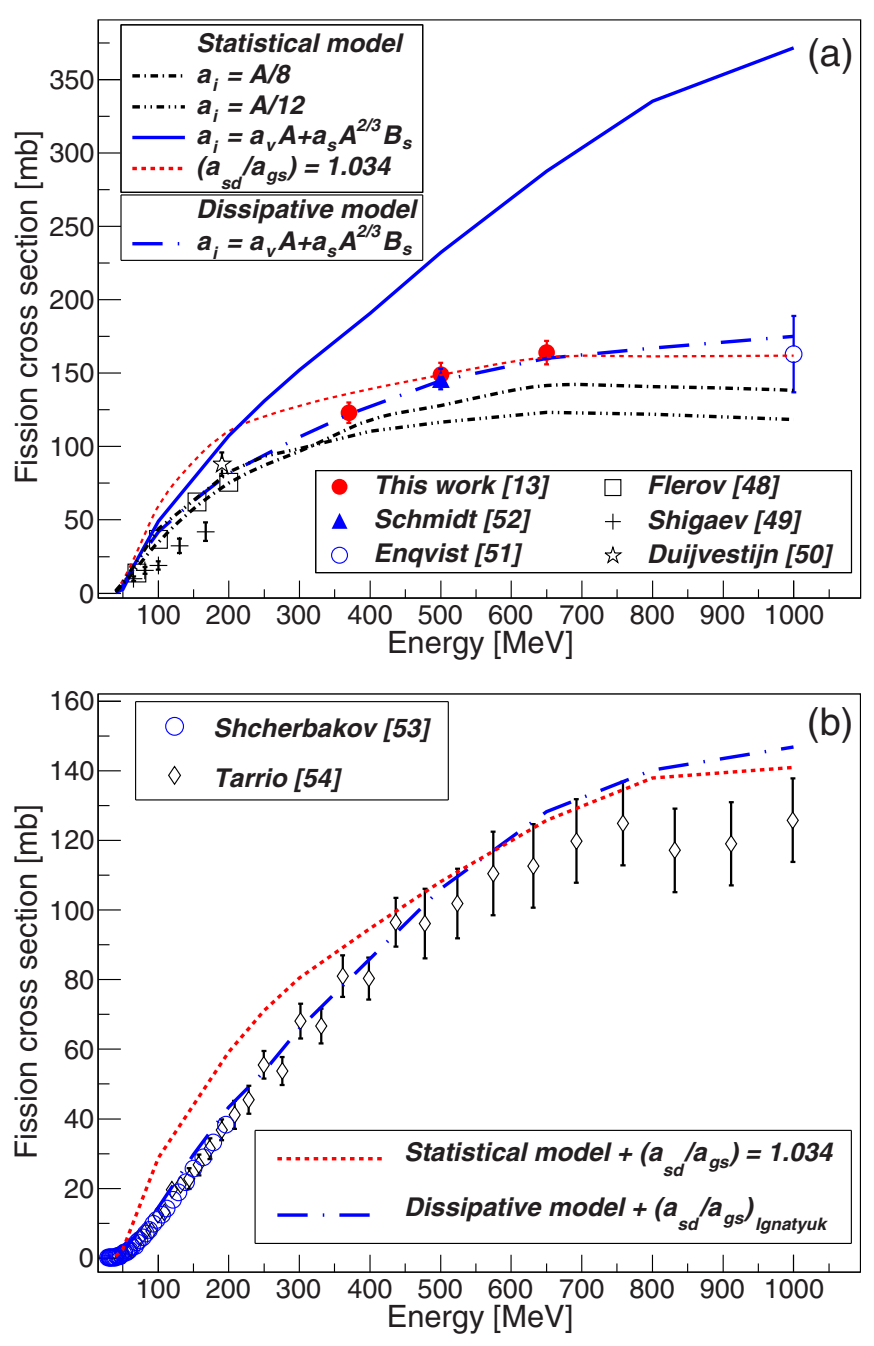

FIG. 1. (Color online) (a) Comparison of the fission cross sections for the reaction ${ }^{208} \mathrm{~Pb}+p$ as a function of the bombarding energy with different model calculations (lines). The data are taken from Refs. [13,48-52]. (b) Same as panel (a) but for the the reaction ${ }^{n a t} \mathrm{~Pb}+n$. The data are taken from Refs. [53,54].

detector permitted us to select fissions produced at the target position [13].

\section{RESULTS AND DISCUSSION}

Total fission cross sections are the most widely used observable for the characterization of the level-density parameter at ground-state and saddle-point deformations [30,35-37]. In our case we employed total cross sections for proton-induced fission reactions on ${ }^{208} \mathrm{~Pb}$ at $370 A, 500 A$, and $650 A \mathrm{MeV}$ obtained in the present experiment [13], as well as previous measurements in direct and inverse kinematics of the same reaction covering kinetic energies up to $1000 \mathrm{MeV}$ [48-52]. This set of data, shown in Fig. 1(a), was completed with the neutron-induced fission cross sections on ${ }^{\text {nat }} \mathrm{Pb}[53,54]$ shown in Fig. 1(b).

The measurements depicted in Fig. 1(a) are overlaid with model calculations using several assumptions to describe the 
level-density parameter. All calculations were done using the code INCL4.6 [55] to describe the first stage of the reaction induced by energetic protons and neutrons according to an intranuclear-cascade model. The resulting remnants were deexcited by using the code ABLA07 [56]. This statistical code describes the emission of $\gamma$ rays, neutrons, light-charged particles, and intermediate-mass fragments (IMFs) according to the Weisskopf formalism [57], which provides a good description of the evaporation residues produced in spallation and fragmentation reactions of nuclei from iron to uranium [29,55,58-62]. The fission decay width is described by using an analytical approximation of the solution of the FokkerPlanck equation describing fission as a diffusion process across the fission barrier [15]. Fission barriers are calculated according to the finite-range liquid-drop model of Sierk [25]. Finally, if fission is reached the mass- and atomic-number distributions of the fission fragments are described by the semiempirical model proposed in Ref. [21] where, according to a statistical picture [63], the width of the atomic-number distribution of the fission fragments is parametrized as a function of the temperature at the saddle point $\left(T_{\mathrm{sd}}\right)$ as

$$
\sigma_{Z}^{2}=\frac{Z_{\mathrm{fiss}}^{2} T_{\mathrm{sd}}}{16 d^{2} V / d v^{2}},
$$

where $d^{2} V / d v^{2}$ is the second derivative of the potential with respect to the mass-asymmetry degree of freedom at the saddle point $v=\left(4 / A_{\text {fiss }}\right) /\left(M-A_{\text {fiss }} / 2\right)$ and is obtained from the systematics of the width of mass distributions measured in Ref. [64]. $A_{\text {fiss }}$ and $Z_{\text {fiss }}$ correspond to the mass and atomic numbers of the fissioning nucleus, respectively, and $M$ represents the mass number of the corresponding fragment. This parametrization was validated in Refs. [21,65-67] by using the mass distributions of the fragments produced in neutron-induced fission on actinides at low energies $[68,69]$ and the charge distributions of the fragments produced in Coulomb-induced fission on several actinides and pre-actinides [70].

Level densities in ABLA07 include intrinsic and collective excitations. Intrinsic excitations are described by using the Fermi-gas formulation [28] with shell [71,72] and pairing $[73,74]$ corrections while contributions from vibrational and rotational levels are formulated according to Ref. [47]. The level-density parameter includes volume and surface dependencies [32,33] parametrized by Ignatyuk and collaborators as

$$
a=a_{v} A+a_{s} A^{2 / 3} B_{s}
$$

where $a_{v}$ and $a_{s}$ are the volume and surface coefficients of the single-particle level densities, respectively, and $B_{s}$ is the ratio between the surface of the deformed nucleus and the surface of the spherical equivalent one taken from Ref. [31]. The values of $a_{v}=0.073 \mathrm{MeV}^{-1}$ and $a_{s}=0.095 \mathrm{MeV}^{-1}$ were taken from Ref. [32].

For the present study, the code ABLA07 was modified in order to calculate fission rates using also the Bohr-Wheeler statistical approach [6] as formulated by Moretto [75]. Moreover, different parametrizations of the level-density parameter were used.
The results of the different calculations for proton-induced fission on ${ }^{208} \mathrm{~Pb}$ are shown in Fig. 1(a). The dot-dashed and double-dot-dashed lines represent statistical model calculations using two deformation-independent parametrizations of the level-density parameters, $A / 8$ and $A / 12$, respectively. These calculations provide a reasonable description of the measured cross sections at low proton energies but clearly underestimate the data for energies above $300 \mathrm{MeV}$. In any case, these parametrizations of the level-density parameter are not realistic because they do not consider modifications due to the different deformations at the ground-state and at saddle-point configurations.

The same statistical model calculation using Ignatyuk's parametrization for the level-density parameter (solid line) shows an overestimation of the data that increases with the proton energy. Finally, the dotted line corresponds to another calculation where the level-density parameter at groundstate deformation ( $\left.a_{\mathrm{g} . s .}\right)$ was obtained using Ignyatyuk's parametrization while the same parameter at saddle-point deformation $\left(a_{\mathrm{sd}}\right)$ was obtained as $a_{\mathrm{sd}} / a_{\mathrm{g} . \mathrm{s} .}=1.034$ following Ref. [35]. One can observe that this calculation describes the data for proton energies above $400 \mathrm{MeV}$ rather well, but it overestimates the data at lower energies. This fact could indicate that the factor between the level-density parameters at the ground state and saddle is not constant with energy due to the difference in the fissioning system for increasing proton energy.

In the same figure, the dot-long-dashed line represents a calculation considering dissipative and transient-time effects with a reduced dissipation parameter $\beta=4.5 \times 10^{21} \mathrm{~s}^{-1}$, according to our previous work [13]. In these calculations the level-density parameter at ground-state and saddle deformations was obtained from Ignatyuk's parametrization. These calculations provide a satisfactory description of the total fission cross sections for the complete range in proton energy covered by Fig. 1(a). The same results were also obtained by Ye and Wang for fusion-fission cross sections of pre-actinides using the Langevin approach [30] and several different descriptions of the level-density parameter. From the comparison of these model calculations with the data, Ye and Wang also found that Ignatyuk's parametrization provides a better description of the evolution of the fusion-fission cross sections with the excitation energy, using a value of (4.0 to 4.5$) \times 10^{21} \mathrm{~s}^{-1}$ for the reduced dissipation parameter.

The same conclusion is obtained when we compare our calculations with the fission cross sections induced by neutrons on ${ }^{\text {nat }} \mathrm{Pb}[53,54]$, as shown in Fig. 1(b). Here we depict only the results of two of the calculations previously discussed, the statistical model calculation with a constant value of the ratio of the level-density parameter at ground-state and at saddle-point deformations $a_{\text {sd }} / a_{\text {g.s. }}=1.034$ (dotted line), and the calculation based on the description of the fission dynamics as a dissipative process using the parametrization of Ignatyuk to determine the value of the level-density parameter at the ground-state and saddle-point deformations (dot-long-dashed line). As observed for proton-induced reactions, the statistical model calculations with a constant ratio for the level-density parameter at the ground-state and at saddle-point deformations overestimate the measured cross sections for neutron 


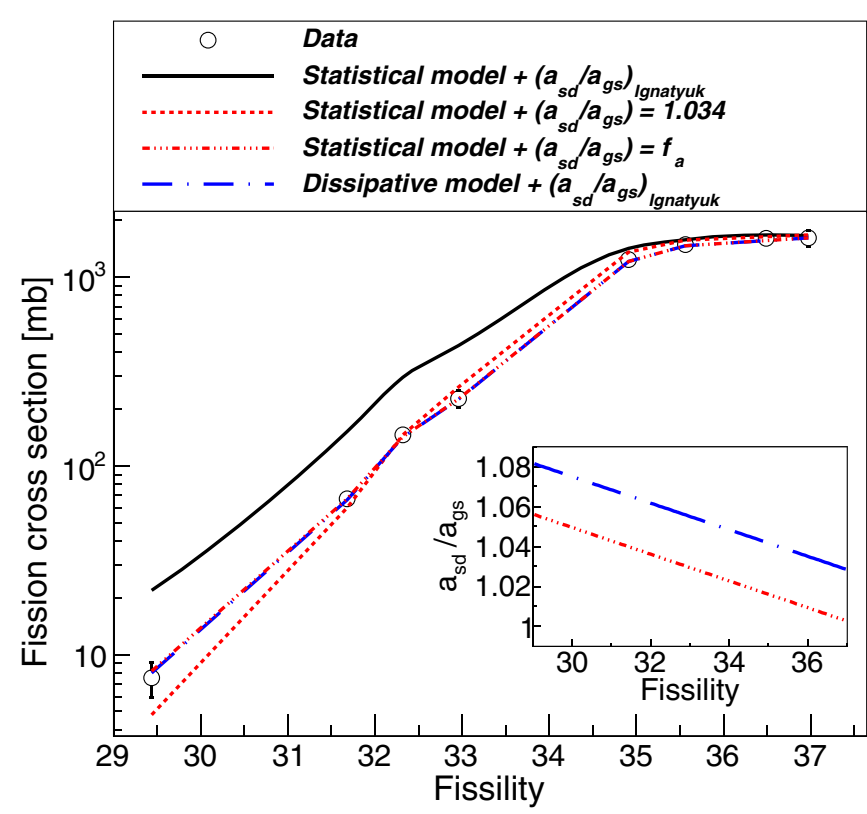

FIG. 2. (Color online) Proton-induced fission cross sections of different nuclei obtained from Refs. [12,13,77] at $500 \mathrm{MeV}$ as a function of the fissility. The lines indicate different model calculations. In the inset we show the ratio $a_{\text {sd }} / a_{\text {g.s. }}$ obtained by our model calculations as a function of the fissility.

energies below $400 \mathrm{MeV}$. The dynamical calculation with a deformation-dependent level-density parameter provides a satisfactory description of the data in this energy range. At higher energies both calculations yield similar results. In this case, the measured neutron-induced fission cross sections seem to be affected by the absence of references for the absolute normalization above $400 \mathrm{MeV}[53,76]$.

To investigate a possible dependence of the ratio of the level-density parameter at ground-state and at saddle-point deformations with the fissility, in Fig. 2 we compare our model calculations with proton-induced fission cross sections obtained for several actinides and pre-actinides between ${ }^{181} \mathrm{Ta}$ and ${ }^{237} \mathrm{~Np}$ at a bombarding energy of $500 \mathrm{MeV}$, taken from Refs. $[12,13,77]$. These data allow one to cover a large range in fissility.

In the figure one can observe that a statistical model calculation with a deformation-dependent description of the level-density parameter based on the parametrization of Ignatyuk (solid line) overestimates the data, while a statistical model calculation with a constant value for the ratio of the level-density parameter at ground-state and at saddle-point deformations $a_{\text {sd }} / a_{\text {g.s. }}=1.034$ (dotted line) underestimates the fission cross sections for nuclei below ${ }^{208} \mathrm{~Pb}$. However, we can describe the fission cross sections by using the same calculation with a fissility-dependent ratio $a_{\text {sd }} / a_{\text {g.s. }}=f_{a}$ (triple-dot-dashed line). On the other hand, we can also describe the data by using a dynamical calculation with a reduced dissipation parameter $\beta=4.5 \times 10^{21} \mathrm{~s}^{-1}$ (dotlong-dashed line). In the inset of the same figure we represent the ratio $a_{\text {sd }} / a_{\text {g.s. }}$ calculated according to Ignatyuk's parametrization (dot-long-dashed line) and the variable ratio

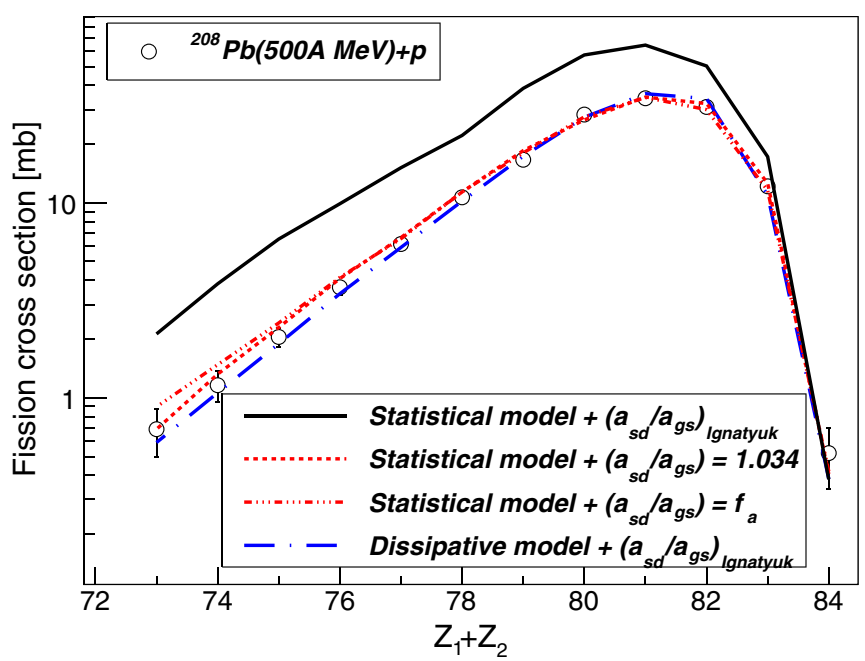

FIG. 3. (Color online) Fission cross section of the reaction ${ }^{208} \mathrm{~Pb}+p$ at $500 \mathrm{~A} \mathrm{MeV}$ as a function of the atomic number of the fissioning nuclei (open circles). The lines represent different model calculations.

$\left(f_{a}\right)$ used in the statistical model calculation (triple-dot-dashed line) as a function of the fissility. The observed offset between the displayed ratios could be related to the reduction in the stationary fission decay width induced by dissipation.

Another observable we propose for this investigation is the evolution of the partial fission cross sections according to the sum of the atomic numbers of the two fission fragments. The sum of the atomic numbers of the two fission fragments $\left(Z_{1}+Z_{2}\right)$ corresponds to the atomic number of the fissioning system, assuming no proton evaporation beyond the saddle point. This assumption is justified because the fission fragments are neutron rich $[42,78]$ and deexcite mainly by neutron emission. This observable shows a strong correlation with the impact parameter and, correspondingly, with the initial excitation energy of the fissioning system $[22,24]$. Therefore, it can be used to constrain model calculations for fissioning systems with different fissilities and excitation energies.

In Fig. 3 we compare the partial fission cross sections of the reaction ${ }^{208} \mathrm{~Pb}(500 \mathrm{~A} \mathrm{MeV})+p$ (open circles) with the model calculations described previously. The partial fission cross sections were obtained by normalizing the yields of the fissioning system [42] to the total fission cross section measured in the present experiment [13]. Calculations based on a statistical description of the fission decay width and using the deformation-dependent parametrization of the level-density parameter proposed by Ignyatuk et al. [32] overestimate the fission cross sections except for the heaviest fissioning nuclei with the lowest excitation energies (solid line) [24]. All other calculations provide a reasonable description of the data. One should notice that this set of calculations includes dynamical and statistical approaches to describe the fission decay width as well as different prescriptions for the level-density parameter. This benchmark indicates that this observable does not show a clear sensitivity to different descriptions of the level-density parameter. 
From these calculations, we could conclude that the fission cross sections can be described by two approaches: a statistical model with a modified ratio $a_{\text {sd }} / a_{\text {g.s. }}$ and a dissipation model with a deformation-dependent ratio $a_{\text {sd }} / a_{\text {g.s. }}$ calculated within Ignatyuk's parametrization. Therefore, total or partial fission cross sections alone cannot be used to disentangle between these two approaches and additional observables are needed.

An observable that was used previously to investigate ground-to-saddle dissipative effects is the width of the charge distribution of the fission fragments. According to Eq. (1), this observable depends on the temperature at the saddle point $\left(T_{\mathrm{sd}}\right)$ which is related to the excitation energy at saddle $\left(E_{\mathrm{sd}}^{*}\right)$ as $T_{\mathrm{sd}}=\sqrt{E_{\mathrm{sd}}^{*} / a_{\mathrm{sd}}}$. Therefore, the width of the atomicnumber distribution could also represent a useful probe to investigate the description of the level-density parameter at the saddle point, providing another important constraint for model calculations.

In Fig. 4(a) we display the width of the atomic-number distribution as a function of the atomic number of the fissioning system $\left(Z_{1}+Z_{2}\right)$ for the different reactions measured in this work: ${ }^{208} \mathrm{~Pb}+p$ at $370 A$ (open crosses), $500 A$ (open circles), and $650 \mathrm{~A} \mathrm{MeV} \mathrm{(open} \mathrm{diamond),} \mathrm{and}{ }^{208} \mathrm{~Pb}+{ }^{27} \mathrm{Al}$ at $500 \mathrm{~A} \mathrm{MeV}$ (open triangles). As can be seen, the width of the atomic-number distribution increases with decreasing $Z_{1}+Z_{2}$, as expected from Eq. (1), if we consider that lighter fissioning systems are produced with higher temperatures. Moreover, for all the reactions we obtain very similar values for the width of each $Z_{1}+Z_{2}$. This result indicates that, for a given fissioning system $\left(Z_{1}+Z_{2}\right)$, the width of the atomic-number distribution of the final fragments depends on neither the entrance channel nor on the bombarding energy of the projectile, supporting then its statistical interpretation. The range in $Z_{1}+Z_{2}$ covered in each reaction is different because it reflects the initial violence of the reaction, and consequently the evaporation probabilities from the increase of excitation energy. Similar conclusions have been pointed out in a recent work [24] using proton- and deuteron-induced fission of ${ }^{208} \mathrm{~Pb}$. Therefore, this observable clearly provides an important constraint for model calculations describing the evolution of the fissioning system from the ground state to the saddle point.

In Fig. 4(b) we compare the data shown in Fig. 4(a) with the different model calculations discussed in this work. The calculations for the reaction ${ }^{208} \mathrm{~Pb}(500 \mathrm{~A} \mathrm{MeV})+{ }^{27} \mathrm{Al}$ were performed with the code ABRABLA07 that consists of the coupling of the abrasion model developed by Gaimard and Schmidt [79] to the deexcitation code ABLA07 [56].

The first conclusion we obtain is that, for the heaviest fissioning nuclei with the lowest excitation energies, there is no sensitivity to the parameters used in the different calculations. The same conclusion was obtained when this observable was used to constrain ground-to-saddle dissipative effects [24]. The reason is that, at low excitation energies, close to the fissioning system $Z_{1}+Z_{2}=82$, the fission delay induced by dissipation is negligible when compared with the statistical time.

For fissioning systems lighter than $Z_{1}+Z_{2}<76$ we observe a sensitivity to the different descriptions of the level-density parameter and the fission decay width. Statistical descriptions of the fission decay width (short-dashed line
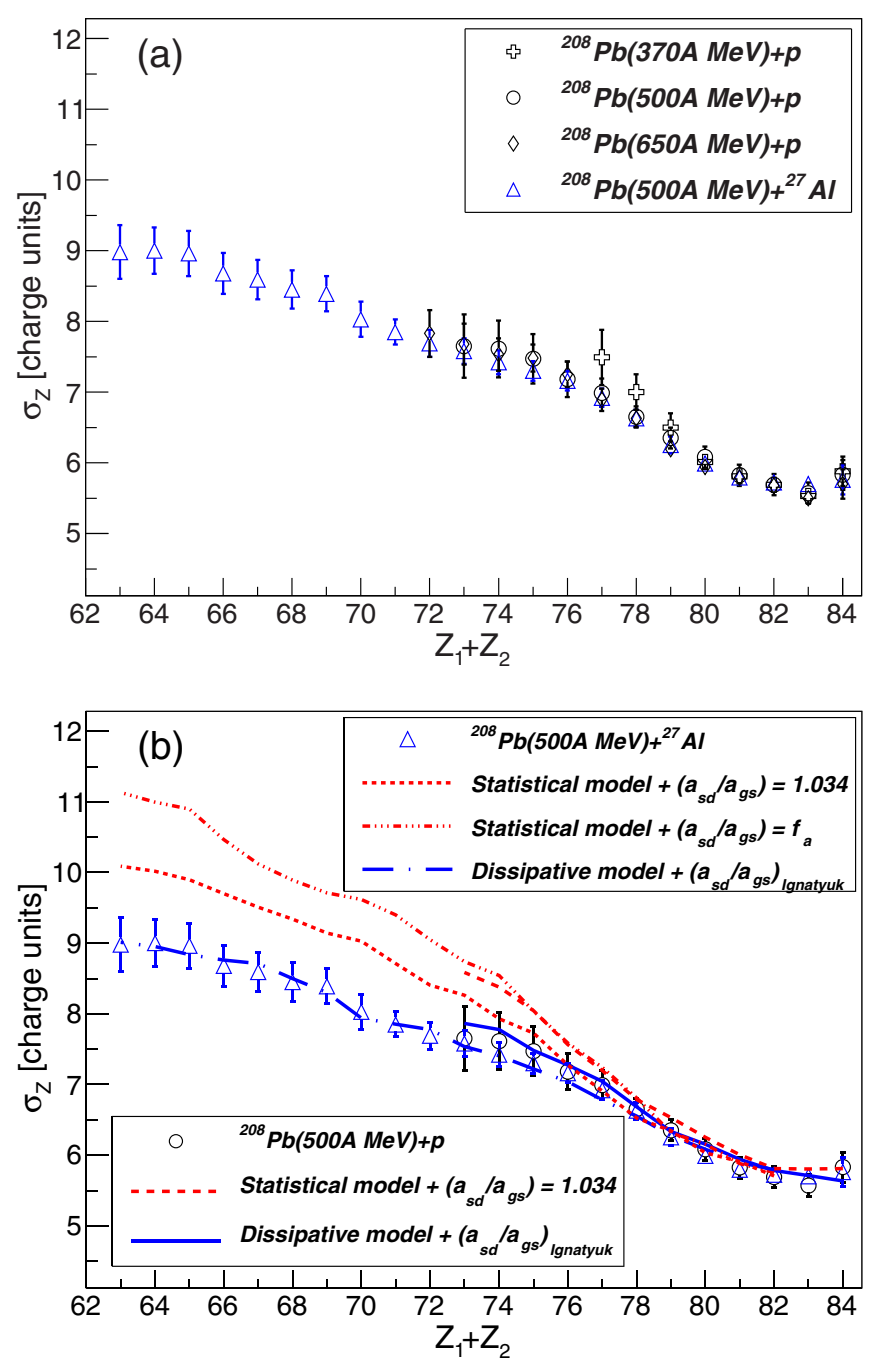

FIG. 4. (Color online) (a) Width of the atomic-number distribution of the final fission fragments measured in spallation and fragmentation reactions of ${ }^{208} \mathrm{~Pb}$ as a function of the atomic number of the fissioning nuclei for different entrance channels and projectile energies. (b) Same as panel (a) but for the data of the reaction ${ }^{208} \mathrm{~Pb}(500 \mathrm{~A} \mathrm{MeV})+{ }^{27} \mathrm{Al}$ (open triangles) and ${ }^{208} \mathrm{~Pb}(500 A \mathrm{MeV})+$ $p$ (open circles). The lines represent different model calculations.

for the reaction ${ }^{208} \mathrm{~Pb}(500 \mathrm{~A} \mathrm{MeV})+p$ and dotted line for the reaction $\left.{ }^{208} \mathrm{~Pb}(500 \mathrm{~A} \mathrm{MeV})+{ }^{27} \mathrm{Al}\right)$ clearly overestimate the width of the charge distributions even when using the phenomenological description of the level-density parameter that reproduces fission cross sections (triple-dot-dashed line). Only calculations considering dissipative effects in the fission decay width and a deformation-dependent level-density parameter following Ignyatuk's parametrization provide a satisfactory description of the width of the charge distributions of the fission fragments [solid line for the reaction ${ }^{208} \mathrm{~Pb}(500 A \mathrm{MeV})+p$ and dot-long-dashed line for the reaction $\left.\left.{ }^{208} \mathrm{~Pb}(500 \mathrm{~A} \mathrm{MeV})+{ }^{27} \mathrm{Al}\right)\right]$, but also of the previous observables discussed in this work.

Similar results and conclusions were obtained in other works $[16,23,24]$, where the authors compared the widths of 
the charge distributions of the fission fragments with statistical and dissipative calculations by using a dynamical description of the level-density ratio based on the parametrization of Ignatyuk. Moreover, those works indicated the need of a dissipative calculation with transient-time effects to achieve a complete description of the data, which is in good agreement with our results. Recently the authors of Ref. [38] also pointed out a similar conclusion when combining the multiplicities of the light-charged particles with fission and evaporation residue cross sections.

\section{SUMMARY AND CONCLUSIONS}

In the present work, we have investigated spallationand fragmentation-induced fission of ${ }^{208} \mathrm{~Pb}$ in inverse kinematics at different bombarding energies by using a highly efficient detection setup that permitted us to measure the atomic number of the two fission fragments in coincidence with good resolution [42]. With these measurements we can determine with high accuracy the total fission cross section as well as partial fission cross sections and the width of the charge distribution of the fission fragments as a function of the sum of the charges of the two fission fragments.

We then used these data to benchmark different model calculations to describe the fission decay width and the level density at ground-state and at saddle-point deformations. In particular, we used a purely statistical description of the fission decay width based on the transition-state method and a dynamical description of the process in terms of a time-dependent fission decay width produced by the coupling between the heat bath describing the excited fissioning nucleus and its collective degrees of freedom through a dissipation parameter. As for the level densities, we used a back-shifted Fermi gas with different descriptions of the level-density parameter; values proportional to the mass number of the nucleus with a constant ratio between the level-density parameters at ground-state and at saddle-point deformations as well as a complete description of this parameter at the saddle and scission configurations by using Ignyatuk's prescription.

The analysis of the reactions proton- and neutron-induced total fission cross sections of ${ }^{208} \mathrm{~Pb}$ and ${ }^{\text {nat }} \mathrm{Pb}$, respectively, as well as partial fission cross sections as a function of the sum of the charges of both fission fragments show that it is possible to combine different models that describe the fission decay width and parametrizations of the level-density parameter to describe the data. This can be understood because we try to describe a multiparametric problem with a single boundary condition. It is then clear that an unambiguous benchmarking of fission models requires several independent observables.

In this work we used the width of the charge distribution of the fission fragments as additional constraint for model calculations. By using these two observables we show that fission of ${ }^{208} \mathrm{~Pb}$ at high excitation energies can only be described properly by using dissipative and transient effects together with a realistic description of the level-density parameter taking into account the deformations of the ground-state and saddle-point configurations.

\section{ACKNOWLEDGMENTS}

The authors are grateful to the GSI accelerator staff for providing an intense and stable beam of ${ }^{208} \mathrm{~Pb}$. We also gratefully acknowledge J.-M. Gheller and S. Leray for providing the liquid-hydrogen target and N. Kurz and A. Prochazka for their technical support during the experiment. This work was partially supported by the European Commission under Projects No. ANDES-FP7-249671 and No. CHANDA-FP7605203, the Spanish Ministry of Research and Innovation under Projects No. FPA2010-22174-C02 and No. ConsoliderCPAN-CSD2007-00042 and the Regional Government of Galicia under the program "Grupos de Referencia Competitiva 2013-011." One of us, C.R.T., also acknowledges the support of the Spanish Ministry of Education under a grant of postdoctoral mobility (2011) administered by FECYT.
[1] J. W. Negele, S. E. Koonin, P. Möller, J. R. Nix, and A. J. Sierk, Phys. Rev. C 17, 1098 (1978).

[2] H. Goutte, J. F. Berger, P. Casoli, and D. Gogny, Phys. Rev. C 71, 024316 (2005).

[3] N. Dubray, H. Goutte, and J. P. Delaroche, Phys. Rev. C 77, 014310 (2008).

[4] G. Scamps and D. Lacroix, Phys. Rev. C 87, 014605 (2013).

[5] G. Scamps and K. Hagino, Phys. Rev. C 91, 044606 (2015).

[6] N. Bohr and J. A. Wheeler, Phys. Rev. 56, 426 (1939).

[7] D. Hilscher and H. Rossner, Ann. Phys. (Paris) 17, 471 (1992).

[8] D. J. Hofman, B. B. Back, I. Diószegi, C. P. Montoya, S. Schadmand, R. Varma, and P. Paul, Phys. Rev. Lett. 72, 470 (1994).

[9] J. P. Lestone, Phys. Rev. Lett. 70, 2245 (1993).

[10] J. Benlliure, E. Casarejos, J. Pereira, and K.-H. Schmidt, Phys. Rev. C 74, 014609 (2006).

[11] J. P. Lestone and S. G. McCalla, Phys. Rev. C 79, 044611 (2009).

[12] Y. Ayyad et al., Phys. Rev. C 89, 054610 (2014).
[13] J. L. Rodríguez-Sánchez et al., Phys. Rev. C 90, 064606 (2014).

[14] M. Thoennessen and G. F. Bertsch, Phys. Rev. Lett. 71, 4303 (1993).

[15] B. Jurado, K.-H. Schmidt, and J. Benlliure, Phys. Lett. B 553, 186 (2003).

[16] B. Jurado, C. Schmitt, K.-H. Schmidt, J. Benlliure, and A. R. Junghans, Nucl. Phys. A 747, 14 (2005).

[17] P. Grangé, Li Jun-Qing, and H. A. Weidenmüller, Phys. Rev. C 27, 2063 (1983).

[18] K. H. Bhatt, P. Grangé, and B. Hiller, Phys. Rev. C 33, 954 (1986)

[19] A. V. Karpov, P. N. Nadtochy, D. V. Vanin, and G. D. Adeev, Phys. Rev. C 63, 054610 (2001).

[20] P. N. Nadtochy, G. D. Adeev, and A. V. Karpov, Phys. Rev. C 65, 064615 (2002).

[21] J. Benlliure, A. Grewe, M. de Jong, K.-H. Schmidt, and S. Zhdanov, Nucl. Phys. A 628, 458 (1998). 
[22] B. Jurado, C. Schmitt, K.-H. Schmidt, J. Benlliure, T. Enqvist, A. R. Junghans, A. Kelić, and F. Rejmund, Phys. Rev. Lett. 93, 072501 (2004).

[23] C. Schmitt, K.-H. Schmidt, A. Kelić, A. Heinz, B. Jurado, and P. N. Nadtochy, Phys. Rev. C 81, 064602 (2010).

[24] Y. Ayyad et al., Phys. Rev. C 91, 034601 (2015).

[25] A. J. Sierk, Phys. Rev. C 33, 2039 (1986).

[26] S. Goriely, M. Samyn, and J. M. Pearson, Phys. Rev. C 75, 064312 (2007).

[27] A. S. Iljinov, M. V. Mebel, N. Bianchi, E. De Sanctis, C. Guaraldo, V. Lucherini, V. Muccifora, E. Polli, A. R. Reolon, and P. Rossi, Nucl. Phys. A 543, 517 (1992).

[28] H. A. Bethe, Phys. Rev. 50, 332 (1936).

[29] J. Benlliure et al., Nucl. Phys. A 700, 469 (2002).

[30] W. Ye and N. Wang, Phys. Rev. C 87, 014610 (2013).

[31] W. D. Myers and W. J. Swiatecki, Ann. Phys. (NY) 84, 186 (1974).

[32] A. V. Ignatyuk, M. G. Itkis, V. N. Okolovich, G. N. Smirenkin, and A. S. Tishin, Yad. Fiz 21, 1185 (1975) [Sov. J. Nucl. Phys. 21, 612 (1975)].

[33] J. Töke and W. J. Swiatecki, Nucl. Phys. A 372, 141 (1981).

[34] W. Reisdorf, Z. Phys. A: At. Nucl. 300, 227 (1981).

[35] D. Mancusi, R. J. Charity, and J. Cugnon, Phys. Rev. C 82, 044610 (2010).

[36] S. Lo Meo, D. Mancusi, C. Massimi, G. Vannini, and A. Ventura, Nucl. Phys. A 933, 43 (2015).

[37] K. X. Jing, L. W. Phair, L. G. Moretto, Th. Rubehn, L. Beaulieu, T. S. Fan, and G. J. Wozniak, Phys. Lett. B 518, 221 (2001).

[38] E. Vardaci et al., Eur. Phys. J. A 43, 127 (2010).

[39] G. Boutoux et al., Phys. Procedia 47, 166 (2013).

[40] T. Gorbinet et al., Phys. Procedia 64, 101 (2015).

[41] J. L. Rodríguez-Sánchez et al., Phys. Procedia 64, 157 (2015).

[42] J. L. Rodríguez-Sánchez et al., Phys. Rev. C 91, 064616 (2015).

[43] B. Voss, Proceedings of the Nuclear Science Symposium and Medical Imaging Conference (NSS/MIC-IEEE, Valencia, Spain, 2011).

[44] E. Pellereau et al., EPJ Web Conf. 62, 06005 (2013).

[45] J. L. Rodríguez-Sánchez et al., EPJ Web Conf. 62, 07009 (2013).

[46] G. Hansen and A. S. Jensen, Nucl. Phys. A 406, 236 (1983).

[47] A. R. Junghans, M. de Jong, H.-G. Clerc, A. V. Ignatyuk, G. A. Kudyaev, and K.-H. Schmidt, Nucl. Phys. A 629, 635 (1998).

[48] G. N. Flerov et al., Sov. At. Energy 33, 1144 (1972).

[49] O. E. Shigaev et al., Khlopin Radiev. Inst., Leningrad Reports, No. 17 (1973).

[50] M. C. Duijvestijn, A. J. Koning, J. P. M. Beijers, A. Ferrari, M. Gastal, J. van Klinken, and R. W. Ostendorf, Phys. Rev. C 59, 776 (1999).

[51] T. Enqvist et al., Nucl. Phys. A 686, 481 (2001).

[52] K.-H. Schmidt et al., Phys. Rev. C 87, 034601 (2013).

[53] O. A. Shcherbakov et al., J. Nucl. Sci. Technol. (Abingdon, U. K.) 39, 230 (2002).
[54] D. Tarrio et al., Phys. Rev. C 83, 044620 (2011).

[55] A. Boudard, J. Cugnon, J.-C. David, S. Leray, and D. Mancusi, Phys. Rev. C 87, 014606 (2013).

[56] A. Kelić, M. V. Ricciardi, and K.-H. Schmidt, in Proceedings of Joint ICTP-IAEA Advanced Workshop on Model Codes for Spallation Reactions, ICTP Trieste, Italy, 4-8 February 2008, edited by D. Filges, S. Leray, Y. Yariv, A. Mengoni, A. Stanculescu, and G. Mank [IAEA INDC(NDS)-530, Vienna, 2008], pp. 181-221.

[57] V. F. Weisskopf and D. H. Ewing, Phys. Rev. 57, 472 (1940)

[58] A. Boudard, J. Cugnon, S. Leray, and C. Volant, Phys. Rev. C 66, 044615 (2002).

[59] M. V. Ricciardi et al., Phys. Rev. C 73, 014607 (2006).

[60] C. Villagrasa-Canton et al., Phys. Rev. C 75, 044603 (2007).

[61] D. Mancusi, A. Boudard, J. Cugnon, J.-C. David, P. Kaitaniemi, and S. Leray, Phys. Rev. C 90, 054602 (2014).

[62] J. Alcántara-Núñez et al., Phys. Rev. C 92, 024607 (2015).

[63] M. G. Itkis, V. N. Okolovich, A. Ya. Rusanov, and G. N. Smirenkin, Sov. J. Part. Nucl. 19, 301 (1988).

[64] A. Ya. Rusanov, M. G. Itkis, and V. N. Okolovic, Phys. At. Nucl. 60, 683 (1997).

[65] A. Kelić, M. V. Ricciardi, and K.-H. Schmidt, in Proceedings of the International Conference on Nuclear Data for Science and Technology, April 22-27, 2007, Nice, France, edited by O. Bersillon, F. Gunsing, E. Bauge, R. Jacqmin, and S. Leray (EDP Sciences, Les Ulis, 2008), pp. 1113-1116.

[66] K.-H. Schmidt et al., Nucl. Phys. A 685, 60 (2001).

[67] K.-H. Schmidt, J. Benlliure, and A. R. Junghans, Nucl. Phys. A 693, 169 (2001).

[68] Ch. Straede, C. Budtz-Jørgensen, and H.-H. Knitter, Nucl. Phys. A 462, 85 (1987).

[69] F. Vives, F.-J. Hambsch, H. Bax, and S. Oberstedt, Nucl. Phys. A 662, 63 (2000).

[70] K.-H. Schmidt et al., Nucl. Phys. A 665, 221 (2000).

[71] A. V. Ignatyuk, G. N. Smirenkin, and A. S. Tishin, Yad. Fiz. 21, 485 (1975) [Sov. J. Nucl. Phys. 21, 255 (1975)].

[72] P. Möller, J. R. Nix, W. D. Myers, and W. J. Swiatecki, At. Data Nucl. Data Tables 59, 185 (1995).

[73] A. V. Ignatyuk, K. K. Istekov, and G. N. Smirenkin, Yad. Fiz. 29, 875 (1979) [Sov. J. Nucl. Phys. 29, 450 (1979)].

[74] A. V. Ignatyuk, M. G. Itkis, V. N. Okolovich, G. R. Ruskina, G. N. Smirenkin, and A. S. Tishin, Yad. Fiz. 25, 25 (1977) [Sov. J. Nucl. Phys. 25, 13 (1977)].

[75] L. G. Moretto, Nucl. Phys. A 247, 211 (1975).

[76] A. D. Carlson et al., Nucl. Data Sheets 110, 3215 (2009).

[77] A. A. Kotov et al., Phys. Rev. C 74, 034605 (2006).

[78] B. Fernández-Domínguez et al., Nucl. Phys. A 747, 227 (2005).

[79] J. J. Gaimard and K.-H. Schmidt, Nucl. Phys. A 531, 709 (1991). 